\title{
Design of tunable metamaterial absorbers based on PIN diodes
}

\author{
Xinxin Xu, Jianjun Jiang ${ }^{a)}$, Ling Miao, Qian Chen, and Biao Sun \\ Intelligent Electronics Institute, School of Optical and Electronic Information, \\ Huazhong University of Science and Technology, Wuhan 430074, China \\ a) jiangjj@mail.hust.edu.cn
}

\begin{abstract}
A tunable metamaterial absorbers (MA) comprised of a new combination shaped metal elements printed on a dielectric substrate loaded with PIN diodes were demonstrated. Through biasing at different voltages to control the diodes, the reflectivity characteristics of the structure can be varied over the entire $2-18 \mathrm{GHz}$ range. A qualitative analysis by a simple visualization method is carried out on the parameters of the tunable MA. Both numerical calculations and microwave measurements have verified the performance.
\end{abstract}

Keywords: tunable, metamaterial absorbers, PIN diodes Classification: Electromagnetic theory

\section{References}

[1] T. Ueda and T. Itoh, "Mu-negative, double-negative, and composite right/ left handed metamaterials based on dielectric resonators," IEICE Electron. Express, vol. 9, no. 2, pp. 65-80, Jan. 2012.

[2] N. Fang, H. Lee, C. Sun, and X. Zhang, "Sub-diffraction-limited optical imaging with a silver superlens," Science, vol. 308, pp. 534-537, April 2005.

[3] D. Schurig, J. J. Mock, B. J. Justice, S. A. Cummer, J. B. Pendry, A. F. Starr, and D. R. Smith, "Metamaterial electromagnetic cloak at microwave frequencies," Science, vol. 314, pp. 977-980, Nov. 2006.

[4] N. I. Landy, S. Sajuyigbe, J. J. Mock, D. R. Smith, and W. J. Padilla, "A perfect metamaterial absorber," Phys. Rev. Lett., vol. 100, pp. 207402-1-6, March 2008.

[5] S. Gu, J. P. Barrett, T. H. Hand, B. I. Popa, and S. A. Cummer, "A broadband low-reflection metamaterial absorber,” J. Appl.Phys., vol. 108, pp. 0649131-6, Sept. 2010.

[6] J. Lee and S. Lim, "Bandwidth-enhanced and polarisation-insensitive metamaterial absorber using double resonance," Electron. Lett., vol. 47, no. 1, pp. 478-479, Jan. 2011.

[7] C. Mias and J. H. Yap, "A varactor-tunable high impedance surface with a resistive-lumped-element biasing grid," IEEE Trans. Antennas Propag., vol. 55, no. 7, pp. 1955-1962, July 2007.

[8] S. Yagitani, K. Katsuda, M. Nojima, Y. Yoshimura, and H. Sugiura, "Imaging radio-frequency power distributions by an EBG absorber," IEICE Trans. Commun., vol. E94-B, no. 8, pp. 2306-2315, Aug. 2011. 


\section{Introduction}

Electromagnetic metamaterials is basically an assembly of identical metallic elements arranged in a one or two-dimensional infinite array with a high frequency selectivity of electromagnetic waves. Up to now, it can be widely used in negative refraction [1], perfect lens [2], invisibility cloak [3], perfect absorber [4] and so on. Recently, many types of MA were demonstrated with high absorption $[5,6]$. However, most of the MA have fixed reflectivity characteristics when it is well-done. Therefore, the tunable MA $[7,8]$ is required to meet the demands of ever changing application. Nevertheless, the tunable band is not wide enough.

In this paper, a tunable MA structure employing new combination shaped metal elements loaded with PIN diodes were presented. Under the guidance of the simple visualization analysis principles, a wide band tunable absorbing response is obtained by adjusting the diode bias voltage of PIN arrays.

\section{Design and performance of tunable MA}

\subsection{Design and calculation}

The MA sheet model is shown in Fig. 1. The absorbing panel consists of a single MA sheet is shown at the insert of Fig. 1(a). It is made of the metal film, thin substrate sheet and a grounded spacer. The overall thickness of the structure is d. The unit cell of MA is an assembly of identical elements arranged on a rectangular lattice. The elements loaded at their centers have been arranged in a rectangular array with inter-element spacing $\mathrm{D}$. The equivalent circuit for the MA is shown in Fig. 1(b). $\mathrm{Y}_{\mathrm{g}}$ denotes the input admittance of the ground plane, $Y_{a}$ denotes the admittance of the freestanding elements alone, $Y_{\text {in }}$ denotes the free-space input admittance of the MA for normal incidence.

The schematic view of composite MA is shown in Fig. 1(c). The PIN diodes are loaded between the combination shaped metal film. The reflectivity responses of the MA can be adjusted by changing the bias voltage of the active PIN diode array. The thickness of the grounded spacer which is adjustable to needs can also change the reflectivity responses. It can be regarded as an assistant cofactor for bias voltage regulation.

\subsubsection{Prediction of the reflectivity by changing $R_{\mathbf{s}}$}

Phillips, BAP70-03 PIN diode is current controlled RF resistor for attenuators. As for the discrete nature of the PIN diode, it can be equal to an adjustable resistor with values of $R_{\mathrm{s}}$ ranging from $10 \mathrm{ohm}$ to 1000 ohm. The change of $R_{s}$ in fact changes the real part of $Y_{a}$ and has little effect on the imaginary part. The analysis process of the tunable MA is shown in Fig. 1.

Fig. 1(c) shows a reflection coefficient of the free-space elements obtained by using the FEM code. In the frequency range of $2 \mathrm{GHZ}$ to $18 \mathrm{GHz}$ one perfect reflection frequency to be around $10 \mathrm{GHz}$ and one null at $15.4 \mathrm{GHz}$ was observed. The admittances of the free-space elements sheet is calculated as

$$
Y_{a}=-2 Y_{0} \Gamma_{1} /\left(1+\Gamma_{1}\right)
$$




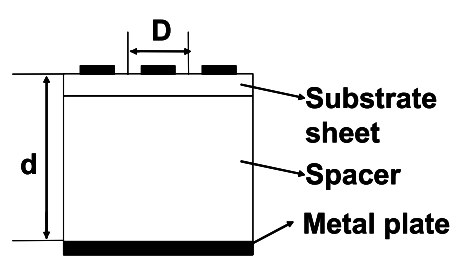

(a)

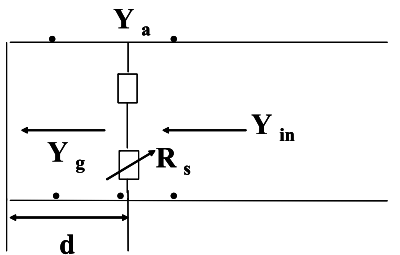

(b)

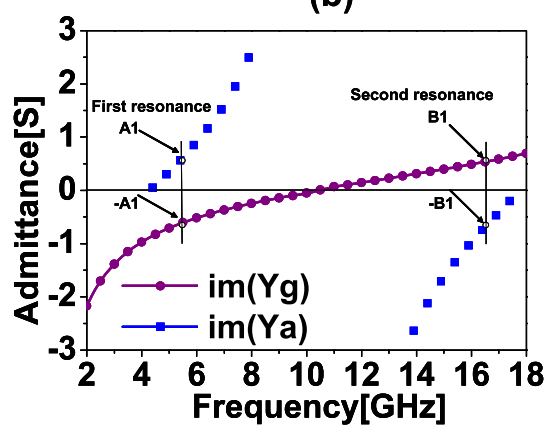

(d) (f)

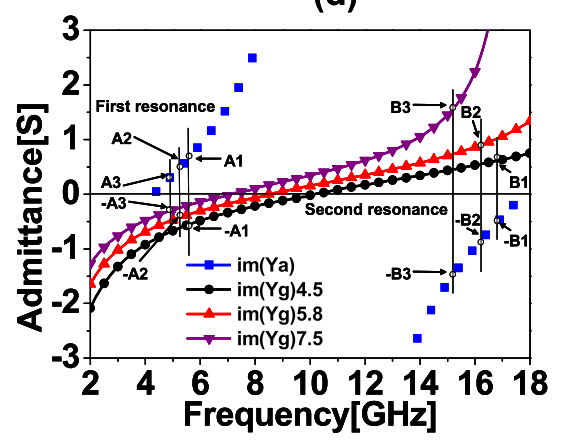

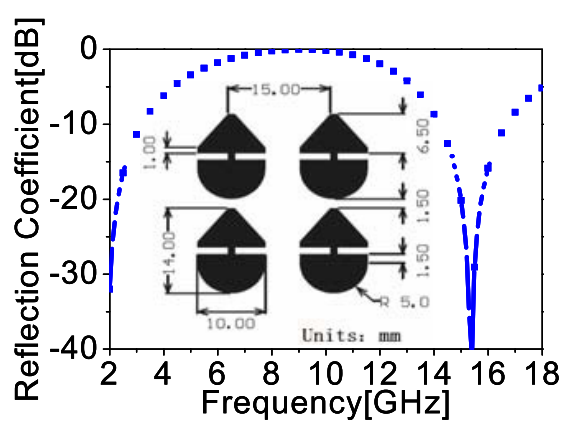

(c)

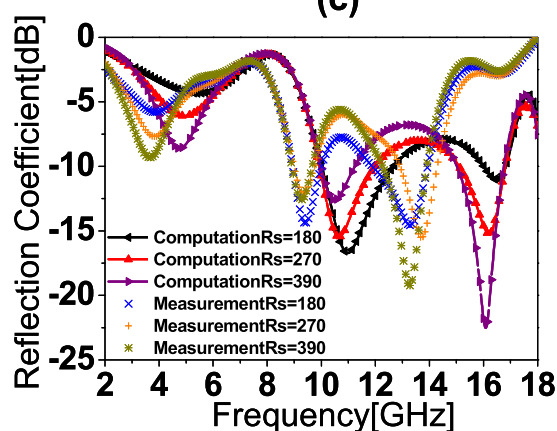

(e)

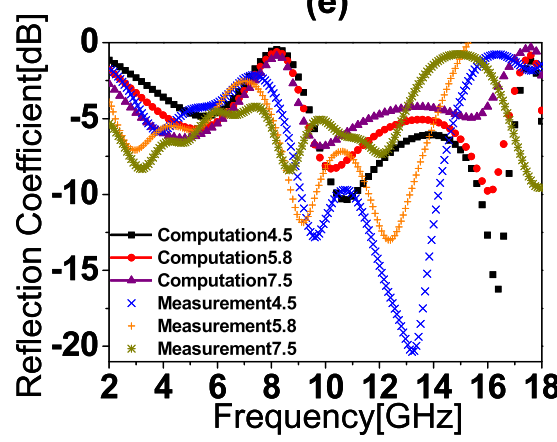

(g)
Fig. 1. The analysis process of the tunable MA (a) Side sketch of MA(b) Equivalent circuit of MA(c) Freestanding elements reflection coefficients(d) Admittances of a $5 \mathrm{~mm}$ grounded slab and free-standing elements(e) Measured Reflection coefficient of the $5 \mathrm{~mm}$ MA for different $\mathrm{R}_{\mathrm{s}}$ compared with the computed result(f) Admittances of grounded slab of different thickness and free-standing elements $(\mathrm{g})$ Measured Reflection coefficient of the MA for different values of d compared with the computed result.

in Fig. 1(d) where $Y_{0}$ represents the admittance of free-space, $\Gamma_{1}$ is the freespace reflection coefficient of the elements which can be computed after a full-wave calculation.

Fig. 1(d) shows the visualization analysis of the admittance match between $Y_{g}$ and $Y_{a}$. The admittance of the $5 \mathrm{~mm}$ grounded dielectric substrate and the admittance of the elements are reported. At the lower frequencies in the band of $2 \mathrm{GHz}$ to $10.4 \mathrm{GHz}$ the value of the imaginary 
part of the input admittance $\mathrm{Y}_{\mathrm{g}}$ of ground plane behaves as an inductor and at high frequencies in the band of $10.4 \mathrm{GHz}$ to $18 \mathrm{GHz}$ behaves as a capacitance. Correspondingly, the elements sheet $\mathrm{Y}_{\mathrm{a}}$ turns from capacitive to inductive behavior in the band of $2 \mathrm{GHz}$ to $18 \mathrm{GHz}$. The elements sheet resonates at $10.4 \mathrm{GHz}$ while the substrate equals acting as a high-impedance wall. Two parallel resonances between the elements and the grounded substrate can be generated at the first resonance around $5.5 \mathrm{GHz}$ and the second resonance $16.7 \mathrm{GHz}$ as shown in Fig. 1(d). Such resonance is obtained when the capacitive admittance of the elements assumes the same value of the inductive admittance of the substrate.

The reflection coefficient of the $5 \mathrm{~mm}$ MA for different $R_{s}$ can be predicted as

$$
\Gamma=20 \log _{10}\left(\left(Y_{0}-Y_{i n}\right) /\left(Y_{0}+Y_{i n}\right)\right)
$$

in Fig. 1(e) where $Y_{i n}=Y_{g}+Y_{a}$ and $Y_{g}=-j Y_{m} \cot \left(2 \pi d \sqrt{\varepsilon_{r}} / \lambda\right)$. From Fig. $1(\mathrm{e})$ it can be seen that the calculated reflectivity characteristics of MA can be varied over the entire $2-18 \mathrm{GHz}$ range, but that there are two "deadspots" at approximately $8 \mathrm{GHz}$ and $18 \mathrm{GHz}$ which are related to the mismatch between $Y_{\text {in }}$ and $Y_{0}$. In the frequency range of $2 \mathrm{GHz}$ to $8 \mathrm{GHz}$, the absorption effect has increased gradually with the increase of $\mathrm{R}_{\mathrm{s}}$. The first absorption peak is located in $5.5 \mathrm{GHz}$ corresponds to the first parallel resonance in Fig. 1(d). In the frequency range of $8 \mathrm{GHz}$ to $18 \mathrm{GHz}$, the performance $-8 \mathrm{~dB}$ in the band from $9.8 \mathrm{GHz}$ to $17 \mathrm{GHz}$ is obtained.

In order to verify the reliability of the calculated result, the PIN diode was replaced by chip resistor. The measured reflection coefficient of three samples realized by different chip resistor value (one declared for $180 \mathrm{ohm}$, another for $270 \mathrm{ohm}$, the third for $390 \mathrm{ohm}$ ) is presented in Fig. 1(e) compared with the computed results. Compared the curves of computed results with that of experiment, the curves changing trend are basically the same. It is seen that there is a difference in the computed and measured peak frequencies. The main reason of this frequency shift should be attributed to the parasitic effect of the chip resistors at high frequencies, in addition to the fabrication error and the coarse soldering process for inserting the chip resistors into the elements. Besides, the finite elements array which is supposed to be infinite at the time of computation.

\subsubsection{Prediction of the reflectivity by changing d}

The change of $d$ in fact changes the imaginary part of $Y_{g}$. The analysis process is shown in Fig. 1.

Fig. 1(f) shows the visualization analysis of the admittance match between $Y_{g}$ and $Y_{a}$. By increasing the thickness of the grounded spacer (from $4.5 \mathrm{~mm}$, to $5.8 \mathrm{~mm}$, and to $7.5 \mathrm{~mm}$ ), the high-impedance wall of the substrate shifts from high frequency to low frequency(from $10.1 \mathrm{GHz}$, to $8.5 \mathrm{GHz}$, and to $7.1 \mathrm{GHz}$ ). As a result, the parallel resonances between the elements and the grounded substrate generated at the first resonance decreases from $5.4 \mathrm{GHz}$ to $4.9 \mathrm{GHz}$ and the second resonance decreases from 16.7 GHz to $15.1 \mathrm{GHz}$.

According to Eq.(2), the reflection coefficient of the MA can be predicted in Fig. $1(\mathrm{~g})$. In the frequency range of $2 \mathrm{GHz}$ to $8 \mathrm{GHz}$, the first absorption peak is moved to the low frequency with the thickness of absorbing material increasing. It is corresponding to the trends of the first

C IEICE 2012

DOI: 10.1587/elex.9.1408 Received August 03, 2012 Accepted August 21, 2012 Published September 12, 2012 
parallel resonance in Fig. 1(f). In the frequency range of $8 \mathrm{GHz}$ to $18 \mathrm{GHz}$, the same performance was obtained which is corresponding to the trends of the second parallel resonance in Fig. 1(f).

The measured reflection coefficient of three samples realized by different $\mathrm{d}$ is also presented in Fig. 1(g) compared with the computed results. Similar with the law in Fig. 1(e), the curves changing trend of the predicted values and measured ones in Fig. 1(g) are basically the same.

\subsection{Fabrication and measurement}

The actual absorption performance of the fabricated tunable MA was measured in an anechoic chamber and compared with the calculated ones. The design example has $11 \times 12$ perfectly conducting elements loaded with one PIN diode (Phillips, BAP70-03) in the middle with a whole size of $180 \mathrm{~mm} \times 180 \mathrm{~mm}$ printed on an FR4 substrate. The whole view and near view photographs of this fabricated MA are shown in Fig. 2(a) and Fig. 2 (b), respectively. The substrate is commercial FR4 with permittivity equal to 4.5 -j0.088 with a thickness of $0.7 \mathrm{~mm}$. The air spacer has been created by employing a homemade foam substrate with a relative permittivity of 1.05j0.017 with a thickness of $4.3 \mathrm{~mm}$.

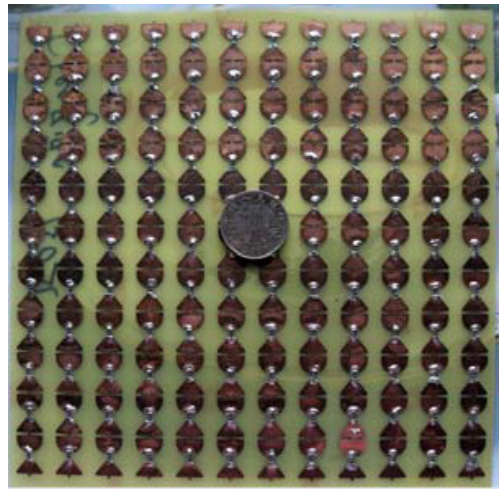

(a)

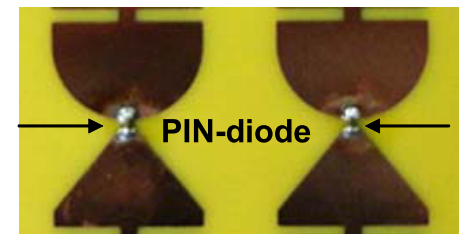

(b)

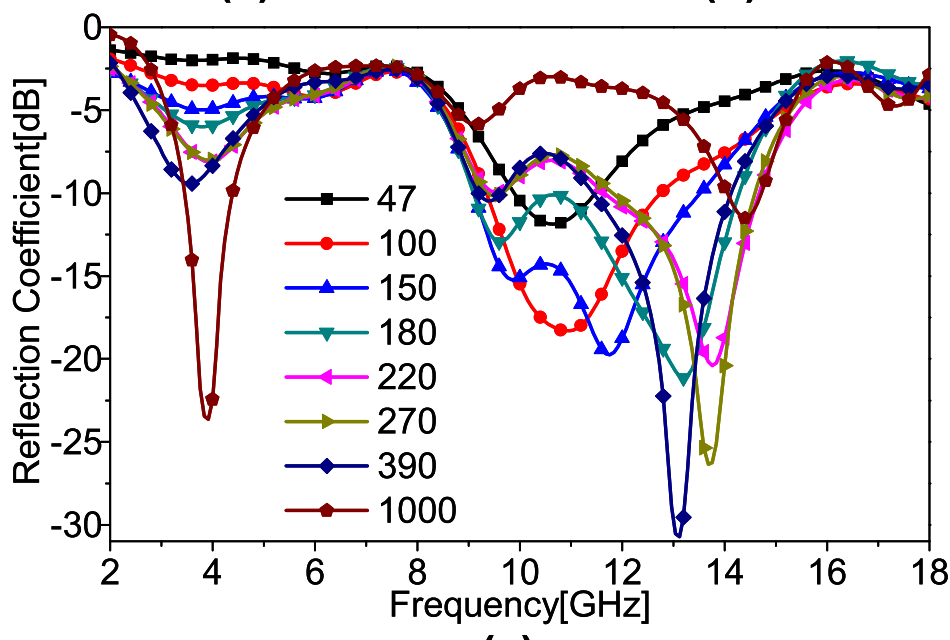

(c)

Fig. 2. Fabrication and measurement of the tunable MA (a) Whole view of MA (b) Near view of MA (c) Measured Reflection coefficient of the $5 \mathrm{~mm}$ MA for different PIN-diode resistance.

\section{(C) IEICE 2012}

DOI: $10.1587 /$ elex.9.1408 Received August 03, 2012 Accepted August 21, 2012 Published September 12, 2012 
The reflectivity characteristics of the tunable MA panel were measured between $2 \mathrm{GHz}$ and $18 \mathrm{GHz}$ in a calibrated NRL arch using an Agilent $82357 \mathrm{E}$ vector network analyzer. For these measurements the MA panel was modulated by a $10 \mathrm{MHz}$ sine-wave signal with $\mathrm{V}_{\mathrm{pp}}=2 \mathrm{~V}$. The MA panel can be controlled by varying the DC bias applied to the Pin diodes.

Fig. 2(c) shows the measured reflectivity responses obtained for various Pin diode resistance levels in the range of $47-1000 \mathrm{ohm}$. Similar with the trends of the test results in Fig. 1(e), there are also two "dead-spots" at approximately $8 \mathrm{GHz}$ and $16 \mathrm{GHz}$. In the frequency range of $2 \mathrm{GHz}$ to $8 \mathrm{GHz}$, the absorption effect has increased gradually with the increase of $\mathrm{R}_{\mathrm{s}}$. In the frequency range of $8 \mathrm{GHz}$ to $18 \mathrm{GHz}$, as the Pin diode resistance is increased from 47 to $100 \mathrm{ohm}$, the reflectivity level reduces across a band of frequencies from 8 to $16 \mathrm{GHz}$ and shows a single absorption peak response at around $4 \mathrm{GHz}$. At the Pin diode resistance of $180 \mathrm{ohm}$ a reflectivity level of less than $-10 \mathrm{~dB}$ is achieved from 9 to $14.5 \mathrm{GHz}$. For further increases in the Pin diode resistance from 150 to $1000 \mathrm{ohm}$, the reflectivity curve shows a double absorption peak response.

\section{Conclusions}

In this paper a deep-depth analysis and design are presented on the reflectivity of tunable MA structure consists of metallic film interconnecting with surface mount PIN diode. Measured results show that the structure can be tuned to provide a variable reflectivity response over a band of frequencies from $2-18 \mathrm{GHz}$. The results demonstrate that the use of the visual graphic method would provide a visualization aid to the transformation of appropriate parameters. These research results provide an effective theory instruction for the design of tunable MA structures for different frequency band usages.

\section{Acknowledgments}

This work has been supported by the National Natural Science Foundation of China (Grant No. 61172003).

\section{IEICE 2012}

